\title{
The Last Diet and Bite
}

\section{Julie Rammal*}

Founder and Owner JSPORT, Holistic Trainer and Practitioner, Fitness Marketing Consultant, USA

*Corresponding Author: Julie Rammal, Founder and Owner JSPORT, Holistic Trainer and Practitioner, Fitness Marketing Consultant, USA.

Received: August 27, 2019; Published: September 06, 2019

Have you ever gone to the supermarket and wandered what are you truly buying? Do you feel confused which diet, or foods to buy? Real foods never lie, and your digestive system is your best friend in deciding. The food industry has evolved to confuse, trick us, and then claim you as a loyal customer. It is hard to really know what to eat, and what not. Let's use: yoghurt, chicken and bread as an example.

A yoghurt is a cultured milk that is thickened and should be filled with protein, probiotics, calcium and should help aid digestion. It is important not to get tempted by the different smells, colours, texture of the available yoghurts. The simple rule is that if it does not fit the true yoghurt definition then it is not really a yoghurt. The most organic source of yoghurt is Greek, goat, sheep milk, soy, and kefir yoghurt. Anything that does fit those descriptions are altered through: colours, texture, smell etc. Don't confuse your taste buds when shopping for a simple yoghurt.

Let's look at chicken. A chicken is a type of domesticated fowl. It is rich in protein, selenium, niacin, and has many health benefits such as: boosts metabolism, heart, natural anti depressant, promotes eye health and bone loss, and helps reduce skin damage. In the supermarket, we are bombarded with so many types of chicken through: labels, sizes, types, colours, texture, smoked, fried and so forth. The best is to always stick to the original source of what you are eating, and just know where is it coming from and how it was raised. Lastly, let's explore bread. Bread is a staple food made from dough of flour, water that is backed. It is the oldest man made food appearing in ancient Egypt in 300 B.C. It is very important for protein, development and repair of the body. However; in the supermarket we have tons of breads to choose from such as: whole wheat, white, multi grain, brown, rye, sourdough and much more. Plus each bread has different packages, lifespan, colours, texture, and can even be completely look, feel, and taste different in another country! Remember, to always shop for fresh baked bread and look for high quality flour and ingredients. Homemade bread are great options.

Regardless of our supermarket experiences, your best friend is your digestive system, that includes your teeth, tongue, liver, pancreas, gallbladder, salivary glands, and the gastrointestinal tract. If any of these are not happy with what you eat, there is a problem. You should explore training your taste buds to know the taste of: astringent, bitter, saltly, pungent, sour, and sweet from natural sources of food. Once you know the taste, you can train your taste buds to sample these 6 tastes on the tongue. Each food should never have too much of one taste. After digestion, you should not experience IBS, bloating, fatigue, nausea, gases, constipation etc...you should just feel normal, and in fact re-energized. If your foods are not providing you this energy, and life force feeling, you need to look at what you are eating and resume to natural organic style of foods that are simple, easily digestible and genuine foods.

Diets are as confusing as the food industry, however; why is something so simple so complicated? To break it down, a diet is a special type of food that restricts someone from. The funny is that, why are so many people constantly on diets and constantly changing them. Animals have their own rhythm of eating, and the only diet they have is when they are sick they restrict themselves from food. However; animals do not have the hundreds of diets that we have such as: Mediterranean, dash, mind, paleo, weight watchers etc. The best diet is to eat natural organic foods that come from earth and have not been altered from their original state. They should not disturb your digestive 
system, mood, or emotions but make you feel re-energized, healthy and happy. These foods are generally low in sugar, high in vegetables and fiber, have few or no refined carbs, no vegatable oils, and have no artificial trans fat.

The best diet, is your own natural diet. Your body will always tell you what it needs if the digestive system was not disturbed. However; since most of us have a unbalanced digestive system, we may not know what to eat. The best way is detox your tongue, fast, and let your body clear all the past taste and food relations that you have had. Once you have cleared everything up, start eating everything that is close to nature such as: vegetables, fruits, nuts, chicken, meat, lamb etc. If you are a vegetarian then honor that by avoiding meats. The further the food is away from earth, the higher chances it is not good for you. For example, a chicken is close to earth, so if left natural it is fine to eat. However; swiss cheese is not close to earth, and has gone through many processes to be altered from its natural state, meaning the chances of gaining weight, cholesterol etc.. can be increased over time.

To summarize, true food does not lie, and does not need to lie. How would you know what is true food? The answer is simple, your entire digestive tract will tell you every time you eat how you feel, your energy, you skin, your hunger levels etc...Food should be simple, organic, fresh and provide a life force to give you everything you need to feel great. If you are still confused, look into detox and fasting methods to reset your digestive system and filter our the bad foods. Your body will naturally design your own diet for you, and if you cant seem to find it start exercising holistically www.jsport.co and you will naturally have your food and taste reprogrammed.

Volume 3 Issue 10 October 2019 (C) All rights are reserved by Julie Rammal. 